\title{
Significant Advances in the Management of Patients with Hematological Disorders, New Challenges and Immense Responsibilities
}

\author{
Kamel Laribi $^{{ }^{*}}$ and Alix Baugier de Materre $^{2}$ \\ ${ }^{1}$ Department of Hematology, Centre Hospitalier du Mans, Le Mans, France \\ ${ }^{2}$ Department of Geriatric, Rothschild Hospital, Paris, France
}

${ }^{*}$ Corresponding author: Kamel Laribi, Department of Hematology, Centre Hospitalier du Mans, Le Mans, France, E-mail: 1-klaribi@ch-lemans.fr

Received Date: March, 2018; Accepted Date: March 18, 2018; Published Date: March 25, 2018

\section{Editorial}

The recent years have seen an acceleration in understanding the physio-pathological mechanisms of many diseases, as well as improvement in their follow-up with more and more powerful tools like flow cytometry, quantitative PCR and more recently the next generation sequencing (NGS), as well as the follow-up of residual disease on liquid biopsies (circulating DNA) but especially the discovery of many new drugs that significantly improved patient's survival.

These new molecules with new mechanisms of action, are very powerful, but also associated with side effects, used most often until disease progression with a considerable cost, open new challenges and raise many questions.

In multiple myeloma, the median survival has doubled in 10 years with new drugs initially used in relapse and then tested in the first line like protease inhibitors (carfilzomib and ixazomib) or anti-CD38 monoclonal antibodies (daratumumab) or SLAMF7 (elotuzumab).

In chronic lymphocytic leukemia, new drugs such as BCR inhibitors (ibrutinib and acalabrutinib) and BCL2 inhibitor (venetoclax) have shown in a number of randomized studies a significant improvement in progression-free survival in patients with relapsed and in some particular forms in the first line. Chemo-free combinations are being tested in the first line to gradually replace chemotherapy. The same is true of other hematological diseases such as Hodgkin's disease or anapalasic lymphoma with anti-CD30 monoclonal antibody (brentuximab vedotin), acute lymphoblastic leukemia with anti-CD3 and anti-CD19 bispecific antibody (blinatumumab).

Even more recently, the arrival of chimeric antigen receptor (CAR) T lymphocytes is a step towards a cure of refractory diseases that failed to respond to several therapeutic lines and that had a very low chance of survival before.

Citation: Laribi K and de Materre AB. Significant Advances in the Management of Patients with Hematological Disorders, New Challenges and Immense Responsibilities. J Clin Cases Rep 2018: 1(1) 8-9. DOI: https://doi.org/10.46619/joccr.2018.1-1003 
www.tridhascholars.org | April-2018

However, all these advances are not without drawbacks: the first is the appearance of new side effects which clinical practitioners are not yet familiar with, as the occurrence of serious infections linked to immunosuppression like aspergillosis, cytokine release syndromes or neuro-toxicities that require the acquisition of new expertise.

These new molecules also raise an economic and ethical problem. These drugs are very expensive, some used until progression for several years. Others cost as CART-cell currently 375000 US dollars for a single cure. Which patient could have access and under which circumstances? At the expanse of what: prevention, treatment of basic diseases such as childhood or infectious diseases?

Unfortunately, not all patients can have access to these new molecules without a strong consensus from all concerned actors: physicians, pharmaceutical companies, health authorities, and the government. 